\title{
Transformaciones en el empleo, transformaciones en el tejido social. Las nuevas clases trabajadoras de servicios
}

\author{
Juan Miguel García Nogueroles \\ Universitat Autònoma de Barcelona. Departament de Sociologia \\ juanmiguel.garcia@campus.uab.cat
}

Recibido: 07-10-2008

Aceptado: 20-03-2009

\section{Resumen}

Las transformaciones acaecidas en el empleo en las últimas décadas nos invitan a considerar la existencia de una nueva clase obrera. En el artículo, se muestran algunos datos que nos llevan a confirmar el proceso de precarización y a suponer el aumento de complejidad en la composición de las clases trabajadoras. La actual situación podría traducirse en la constitución de una nueva clase trabajadora de servicios, siempre y cuando se consiga superar las dificultades de identificación y de toma de consciencia derivadas de la realidad de la multisegmentación de los mercados de trabajo, que fragmenta las sociedades.

Palabras clave: trabajo, precarización, segmentación, clase social, empleo.

Abstract. Employment changes and social transformations. The new services' working classes

Changes in employment in the last decades suggest the emergence of a new working class. This article presents some data that show a process of increasing precariousness and complexity in working classes' composition. The present situation might lead to the constitution of a new service working class, at least if this new class is capable of overcoming selfidentification and self-consciousness problems, which are produced by the segmentation of the labour market and the fragmentation of societies.

Key words: work, increasing precariousness, segmentation, social class, employment.

\section{Sumario}

1. Transformaciones en el empleo

2. Las nuevas clases trabajadoras. Trabajadores y ciudadanos precarios
3. Conclusiones. Nuevas clases trabajadoras, nueva estructura social

4. Bibliografía 


\section{Transformaciones en el empleo}

Actualmente, el empleo en el mercado está experimentando dificultades contundentes para alcanzar el grado de centralidad en la vida de los individuos del que disfrutaba hacia el final de la década de los setenta. La emergencia de la precariedad en las relaciones laborales ${ }^{1}$ hace que la llamada norma social del empleo fordista ${ }^{2}$ se haya disuelto hasta desaparecer entre las nuevas políticas de la flexibilidad, el actual episodio de segmentación de los mercados de trabajo y el proceso de fragmentación social que se padece en los países desarrollados de occidente y en aquéllos, en vías de desarrollo, que participan del modelo global de producción y distribución capitalista.

En definitiva, el escenario de las relaciones laborales a partir de la década de los setenta presenta una compleja situación a partir de la pérdida de la seguridad en el empleo (Miguélez, 2006 y 2007) o, al menos, en los empleos de los segmentos laborales más precarios. Esta precarización —en cuanto a flexibilidad e inseguridad en los empleos y en las carreras laborales, así como en los ingresos y en las condiciones objetivas de trabajo- es especialmente grave en tanto la situación inmediatamente anterior se caracterizaba precisamente por una mejora continua y acumulable de las condiciones de trabajo y la seguridad laboral (Miguélez, 2002). De esta forma, debido a la precarización, el número de ciudadanos que se encuentran desprotegidos frente a las contingencias de las economías de mercado va en aumento, ya que el hecho de que el empleo haya abandonado su fuerza normativa no es óbice para que conserve, todavía, todo su potencial como principal generador de rentas en una sociedad que deja de ser, nominalmente, del trabajo, aunque sin haber abandonado a éste - más bien a su versión visible en el mercado, el empleo- como base principal de la economía familiar, de sus recursos para el consumo y, también, como elemento fundamental de la recaudación tributaria y, a través de ésta, como generador de derechos sociales más o menos tangibles.

Las diversas perspectivas teóricas que se han fijado en este proceso de transformación de las relaciones laborales a lo largo de las últimas tres décadas, ya desde Braverman (1974), muestran una profunda preocupación por las distintas divisiones que se establecen entre los trabajadores. Las teorías de la segmentación ${ }^{3}$ ofrecen un amplio análisis en este sentido y, además de los mecanismos conocidos de esta segmentación, podemos establecer que la desregulación, la búsqueda de la flexibilidad en el trabajador, las nuevas formas de organización de la producción - formas flexibles o ligeras frente a la rigidez fordista-, el proceso de racionalización productiva (Kern y Schumann, 1988) o las nuevas formas de colaboración entre empresas y de dirección de

1. Con las llamadas formas atípicas de empleo (Marshall, 1992).

2. Por ejemplo como la define Castel (Castel, 1997). Para el empleo como norma social, es interesante consultar Prieto (2007).

3. Véanse Gordon, Edwards y Reich (1986) y Villa (1990). Para el caso español, y en cuanto a la construcción de tipologías, son interesantes las investigaciones de López Roldán (1996) y de López Roldán et al. (1998). 
éstas (Castells, 1997; Bueno Campos, 2002) están permitidas, también, por la revolución tecnológica y los avances en materia de gestión y organización de la producción que esta tecnología ha facilitado. Por lo tanto, todos estos son factores que deben ser considerados de manera exhaustiva si se pretende realizar un análisis correcto de la situación de las clases trabajadoras en nuestros días.

\section{Las nuevas clases trabajadoras. Trabajadores y ciudadanos precarios ${ }^{4}$}

Como ya se ha apuntado, sabemos que el empleo resulta ser una fuente importantísima de derechos sociales a pesar del actual adelgazamiento del estado de bienestar. Que a partir del empleo se puede alcanzar el estatus pleno de ciudadano en las sociedades occidentales no significa que aquel que no trabaja no tenga derechos civiles o no sea considerado ciudadano, pero lo cierto es que todavía pesa en estas sociedades el hecho de que trabajar es lo normal y que, así, todo el mundo puede disponer de un empleo, de manera que muchas de las prerrogativas y servicios del estado de bienestar se ofrecen a través del trabajo, de la actividad productiva en el mercado, puesto que disponer de ésta es lo que se considera habitual.

Desde este punto de vista, el ciudadano es también un trabajador y el trabajador, un ciudadano, y quien no trabaja en el mercado lo hace en tareas reproductivas que facilitan, precisamente, la reproducción de este trabajo en el mercado o está en una situación —enfermedad, desempleo- que espera resolver pronto. Aquél que trabaja, además, dispone de rentas suficientes para realizarse como persona - como ciudadano- en el contexto social en el que vive, $y$ es impensable que alguien que desarrolle una tarea laboral se encuentre en una situación donde, por falta de ingresos, vea comprometida su ciudadanía. Porque ser ciudadano se ha convertido, igualmente, en ser consumidor (Bauman, 2001), y no alcanzar unos determinados mínimos de consumo o de capacidad de éste produce de manera automática situaciones de exclusión social. Pero, en nuestras sociedades modernas, empieza a ser habitual la figura del trabajador que, a pesar de desarrollar un trabajo remunerado, incluso a tiempo completo, no alcanza los ingresos mínimos para conseguir una correcta inserción social. El concepto anglosajón de working poor puede ser una buena piedra de toque para discutir sobre este fenómeno (Medialdea y Álvarez, 2005), aunque - a parte de la dimensión económica - también se debe tener en cuenta en la definición del concepto un elemento de cariz social que se refiere a las unidades familiares y sus estrategias de supervivencia en las estructuras sociales y comunitarias en las que se ven inseridas (EUROFUND, 2004), y los efectos de las distintas realidades sociales a la hora de caer o no en esta situación determinada (US Department of Labor, 2006).

4. Algunas de las ideas desarrolladas en este epígrafe han sido planteadas en García Nogueroles (2009). 
Lo cierto es que la ruptura de la norma social del empleo fordista y la substitución por la nueva situación donde lo normal es, precisamente, el empleo flexible, ha acabado con la idea de que el empleo debe retribuir suficientemente al trabajador. El salario se convierte ahora en un producto resultante de las leyes de la oferta y la demanda en un momento en el que las capacidades de negociación de los trabajadores y la existencia de un importante ejército de reserva - así como las presiones hacia la moderación salarial, encarnadas, por ejemplo, en la lucha contra la inflación- dificultan bastante que los salarios, en especial en los empleos menos cualificados y con menores productividades, puedan crecer.

Una parte importante de estos trabajadores precarios son las nuevas clases trabajadoras que, a diferencia de otras etapas anteriores, en una proporción importante, encuentran su puesto de trabajo en los servicios; pero estos nuevos trabajadores sufren una situación de indeterminación respecto al tipo de empleo que podrán desarrollar en el futuro o a si podrán, realmente, estar ocupados. La falta de empleo tiene una consecuencia determinante sobre la vida del trabajador, ya que lleva asociada una falta de ingresos y, por tanto, como ya se ha apuntado, de capacidad de consumo. Es complicado señalar cuales son los ingresos mínimos (Recio, 2001) que un trabajador o una unidad familiar ha de alcanzar para no sufrir un proceso de exclusión social, ya que dependerá de multitud de factores, pero sí que debemos considerar que, por debajo de un determinado nivel de ingresos, esta persona o unidad familiar empezará a sufrir el proceso de exclusión. La respuesta del trabajador frente a esta situación pasa por encontrar pronto un empleo -o un mejor empleo-, un lugar donde poder vender su fuerza de trabajo a cambio del salario necesario; pero siendo el trabajador quien debe encontrar el empleo, sus posibilidades de negociar las condiciones de estas actividades son muy reducidas ya desde el inicio de la búsqueda, especialmente si el trabajador no dispone de otras fuentes de ingresos - prestaciones sociales, familia, ahorros- que le permitan estar un tiempo buscando el trabajo y escogiendo la mejor opción ${ }^{5}$.

En este sentido, hay que considerar que las oportunidades de empleo, como venimos comentando, se encuentran principalmente en los sectores terciarios (tabla 1), de forma que será en estas actividades en las que los individuos podrán encontrar su puesto de trabajo. Estas oportunidades de empleo, en gran medida, van a estar determinadas por las necesidades existentes de determinados servicios a las empresas y a las personas donde la cualificación profesional que se precisa no es muy elevada y que constituyen la base de las economías postindustriales, base sobre la que se pueden desarrollar todas estas otras actividades, muchas también terciarias y relacionadas con la gestión de la información y la comunicación, que sí cuentan con elevadas productividades y cuyos salarios y condiciones generales de empleo serán más favorables. Y un rasgo importante de estos nuevos empleos de servicios poco cualificados y de baja

5. Este hecho se discute ampliamente, entre otras problemáticas, en Bilbao (1999). 
Tabla 1. Tasa de ocupados según el sector productivo en algunos países (2007)

\begin{tabular}{lllcl}
\hline & Agricultura & Industria y construcción & Construcción & Servicios \\
\hline UE 27 & 5,57 & 27,53 & 8,15 & 66,39 \\
UE 25 & 4,46 & 27,23 & 8,18 & 67,77 \\
UE 15 & 3,47 & 26,17 & 8,18 & 69,73 \\
Alemania & 2,25 & 29,82 & 6,62 & 67,93 \\
España & 4,55 & 29,27 & 13,25 & 66,18 \\
Francia & 3,36 & 22,76 & 6,72 & 71,68 \\
Italia & 3,98 & 30,16 & 8,42 & 65,86 \\
Reino Unido & 1,36 & 22,01 & 8,15 & 76,29 \\
\hline
\end{tabular}

Fuente: datos de EUROSTAT, consultados en INE (2009) y elaboración propia.

productividad es que podrían estar siendo ocupados mayoritariamente por trabajadores de colectivos muy determinados - mujeres, jóvenes, inmigrantes-, lo cual incide en la desigualdad de oportunidades para los trabajadores $\mathrm{y}$ en la consolidación de determinados grupos en los segmentos secundarios del mercado de trabajo.

Así, en definitiva, las nuevas clases trabajadoras, mayoritariamente de servicios, podrían ser una reelaboración de las anteriores clases trabajadoras, un aglutinamiento en base social de los segmentos secundarios del mercado laboral. Su propia existencia demostraría que los cambios habidos en el trabajo, en las maneras de trabajar y en las maneras de estar contratado, inciden directamente en el tejido social, lo modifican, lo alteran y producen nuevas expresiones sociales que se construyen y se destruyen en relación con el resto de mundos comunitarios. Para definir a esta clase trabajadora actual ya no sería útil la distinción entre propietarios y no propietarios de los medios de producción. Tampoco sería útil hablar de consciencia de clase cuando todavía se está en los inicios de la constitución objetiva de esta nueva colectividad obrera. Para definirla utilizaremos, por un lado, las teorías de la segmentación y la fragmentación social y, por el otro, la realidad del proceso de precarización del empleo. De esta manera, podremos tener en cuenta su existencia sólo en relación con las condiciones objetivas de empleo que se dan actualmente, así como con las vivencias de los trabajadores respecto a este empleo; considerando, como se ha apuntado, especialmente el empleo que se genera en los servicios, en concreto, en determinados subsectores como la hostelería, el comercio, los servicios a las personas y algunos servicios a las empresas, como puede ser el caso, por ejemplo, de la limpieza.

En resumen, se ha señalado que las nuevas clases trabajadoras existen entre los segmentos más débiles de los mercados de trabajo. Para confirmar esta existencia deben darse, por un lado, condiciones objetivas de precariedad en el empleo y, por el otro, condiciones de tipo social y comunitario que producen 
que estas clases incluyan grupos y colectividades más o menos definidos. Con la cuestión social superada para la mayor parte de las sociedades occidentales el espacio de lucha de estas nuevas clases obreras es otro. No será tanto la supervivencia y la reproducción de la fuerza de trabajo como asegurar unas condiciones de trabajo que permitan desarrollar una vida plena y satisfactoria; que permitan participar en igualdad de condiciones en los mercados de trabajo; que permitan estar presentes en la vida política y comunitaria, en la sociedad civil; etc.

Al mismo tiempo, las nuevas clases trabajadoras podrían no existir únicamente a partir de la constatación de la presencia en los mercados laborales de colectivos más débiles que el resto, ya que esta situación se ha dado siempre, desde las primeras divisiones del trabajo y, por supuesto, desde el inicio de la edad salarial que podemos fechar hacia el comienzo del siglo XIX, con el desarrollo de la revolución industrial. Y, precisamente, aquello que permitía hablar de la existencia del proletariado como clase o grupo social durante el siglo XIX será lo que nos permita hablar de la nueva clase obrera de servicios a día de hoy: y esto es el incremento real de sus efectivos y el incremento de la importancia relativa de estos segmentos de población — fragmentos, en realidad- en la constitución de las realidades sociales y comunitarias. En efecto, el número de empleos que dan acceso a salarios modestos está creciendo ${ }^{6}$ de una manera significativa, y aquel escenario del futuro del trabajo que indicaba que las necesidades de formación serían cada vez mayores para los trabajadores en una sociedad muy tecnologizada, que exigiría de sus activos un continuo reciclaje y múltiples entradas y salidas del mercado de trabajo con este fin —en un ejer-

6. En el caso de España, con datos de la última década — desde el final del segundo trimestre de 1996 hasta el final del segundo trimestre de 2007, según datos de la EPA consultados en INE (2007)—, se observa que algunos de los sectores productivos que, de manera más espectacular - con un incremento cercano al 100\% de los efectivos e incluso superior en la categoría de hogares que emplean personal doméstico y en el sector de la construcción-, han aumentado su número de activos, han sido la hostelería, que pasa de 770.000 empleados a casi rozar el millón y medio; las actividades sociales, los servicios prestados a la comunidad y los servicios personales, que pasan de 470.000 empleados a los 850.000; los hogares que emplean personal doméstico, que pasan de 370.000 a más de 760.000 , y la construcción, que pasa de un millón doscientos mil trabajadores a dos millones setecientos mil, aunque tendríamos que valorar la destrucción de empleo que, durante 2008, se ha generado en este sector. Otras actividades, como el comercio —que incluye también la reparación de vehículos-, tienen también incrementos muy importantes de trabajadores y, así, pasan de los dos a los tres millones de activos, pero el incremento en este sector debe ser incluido dentro de la dinámica normal de creación de empleo que ha sido típica de este periodo. Es importante señalar que el sector que crece de manera más rotunda es uno que, en principio, no está marcado por características como la falta de cualificación o la precariedad. Así, el sector de actividades inmobiliarias y de alquiler y de servicios empresariales pasa de 760.000 trabajadores a los dos millones de activos en 2007, posiblemente de acuerdo con la expansión del sector inmobiliario y de las actividades de promoción y compraventa de viviendas y en completa relación con el incremento de trabajadores en la construcción, si bien habrá que considerar una desaceleración de este crecimiento durante 2008, fruto de las dificultades económicas y financieras que se consolidan durante este año. 
cicio de flexibilidad positiva de los mercados laborales, que combinarían etapas de actividad con otras de cualificación-, se reduce a un porcentaje muy pequeño de los trabajadores: aquéllos de los segmentos primarios independientes o aquéllos, todavía jóvenes, que aspiran a encontrar un empleo de calidad y que viven la flexibilidad como un camino necesario hacia unos buenos ingresos y un empleo cualificado. La realidad es que el trabajador de los segmentos inferiores no precisa altos niveles de formación en nuevas tecnologías o procesos de trabajo, porque, precisamente, esta baja necesidad de capital humano es lo que permite su fácil intercambio sin que las medidas y tasas de calidad global de los servicios se vean sensiblemente afectadas.

Hablar de la nueva clase trabajadora de servicios es, en definitiva, hablar de trabajadores precarios, con salarios bajos, con una fuerte inestabilidad fruto de la temporalidad en el empleo, con muchas dificultades de articular un discurso de protesta o de descontento más allá de las quejas individuales hacia su situación concreta. Y, como tal, la investigación respecto a esta clase social debería basarse, en primer lugar, en una revisión de las principales dimensiones respecto a las relaciones laborales que permita, así, realizar una valoración de la calidad en el empleo, tanto de manera global como para aquellos grupos que, desde las distintas perspectivas teóricas, han sido señalados como destinatarios de condiciones de trabajo más duras.

\section{2a. Evidencias de la precarización en la UE}

En el contexto de la Unión Europea, los datos muestran que el grado de satisfacción de los trabajadores con su empleo es realmente elevado, pero es importante señalar que existen muchas diferencias entre los distintos países que componen la Unión Europea - diferencias que han crecido con la última ampliación que ha dejado en 27 el número de países miembros- y entre los grupos sociales y las condiciones en las que éstos son capaces de acceder al empleo. En este sentido, distintas publicaciones fruto de los resultados que ofrecen los estudios estadísticos de la Comisión Europea señalan un ligero empeoramiento de las condiciones laborales de los trabajadores europeos, en general, así como la existencia, entre algunas colectividades sociales, de peores condiciones de trabajo que la media. Así, en los últimos años, se detecta un ligero pero constante incremento en la contratación temporal en Europa que la deja en torno a valores (EUROFUND, 2005) del 15\% y que afecta más a las mujeres que a los hombres y, de forma muy especial (EUROFUND, 2007), a los jóvenes, donde los contratos temporales incluso superan el $40 \%$ de los totales. En el caso de los contratos a tiempo parcial, éstos también están experimentando un ligero incremento, en especial a partir de 2002, de manera que se alcanzan valores cercanos al $20 \%$ del total de las contrataciones. Las diferencias entre hombres y mujeres sí son en este caso más sensibles y son las mujeres las que se quedan con la mayor parte de los contratos a tiempo parcial (EUROFUND, 2007), de manera que tres de cada diez mujeres en Europa trabajaban a tiempo parcial en 2005 frente a, únicamente, un $7 \%$ de los hombres. Ellas, ade- 
más, se encuentran más frecuentemente en situaciones de desempleo y en empleos con salarios bajos (EUROFUND, 2004), de manera que los ingresos de una mujer o de un hogar donde la mujer sea la fuente principal de ingresos pueden verse interrumpidos con mayor frecuencia y, por lo tanto, hacer caer a ésta en alguna situación asimilable a la exclusión.

En cuanto a condiciones de trabajo, los datos muestran resultados muy optimistas, siempre por encima del $50 \%$ de respuestas positivas, en las variables referentes a la autonomía de los trabajadores en sus puestos de trabajo (EUROFUND, 2005), pero en todos los casos hay un descenso en estos indicadores que está entre el 2 y el 5\%, si consideramos los quince años entre 1991 y 2005. En cuanto a los indicadores que se refieren al contenido de los empleos, otra vez los resultados vuelven a ser bastante positivos, por encima del $70 \%$ de respuestas positivas en los diferentes indicadores, pero vuelve a detectarse un descenso de aproximadamente un 5\% en todos ellos. Hay que tener en cuenta, además, que hasta un $43 \%$ de los europeos consideran que su trabajo implica realizar tareas monótonas, y aunque la satisfacción de los europeos con su empleo es muy alta, hay otro descenso en la calificación global de esta variable si la observamos en perspectiva temporal. En definitiva, a pesar de que los datos son buenos, la tendencia de éstos es hacia el empeoramiento.

Se detectan diferencias salariales muy importantes entre hombres y mujeres (EUROFUND, 2005), ya que, aunque las tasas de ocupación femenina han aumentado, también lo han hecho las tasas de paro femeninas y, en el mismo sentido, su presencia entre las posiciones de responsabilidad, mando o muy cualificadas es todavía bastante inferior a la de los hombres. En definitiva, la diferencia salarial, o gender gap - que se encuentra sobre el 15\% del salario-, entre hombres y mujeres se mantiene inamovible, en los últimos años, para el caso de la Unión Europea. Hay que tener en cuenta que, en general, son más las mujeres que los hombres las que, de nuevo en los países de la Unión Europea, perciben el salario mínimo, aunque estos salarios mínimos (Regnard, 2007) son muy desiguales si consideramos todos los países de la Unión, puesto que van desde los poco más de 90 euros de Bulgaria a los más de 1.500 de Luxemburgo.

\section{2.b. Evidencias de la precarización en España}

Para el caso español ${ }^{7}$ (INE, 2004), es importante señalar que las mujeres también se encuentran peor pagadas y son mayoría - para el año 2002- entre los trabajadores que perciben sueldos menores de 10.500 euros anuales $y$, al mismo tiempo, no se encuentran significativamente presentes entre los que perciben más de 50.000, mientras que, a partir de esta cifra, todavía se puede localizar un importante contingente de hombres. En cuanto al tipo de con-

7. La media (INE, 2004) se encontraba, en 2002, alrededor de los 19.000 euros anuales, pero la moda de la distribución —el salario percibido por un mayor número de trabajadoresestá, únicamente, en torno a los 12.000 . 
trato, edad y nacionalidad, sabemos que aquéllos que disponen de un contrato de duración determinada llegan a ganar hasta un $40 \%$ menos que los que trabajan con un contrato indefinido, porcentaje que llega hasta el $44 \%$ cuando el trabajador es mujer (INE, 2004: 16-17). En cuanto a la edad, se detecta un aumento de las retribuciones salariales al mismo tiempo que aumenta la edad del trabajador, cosa que no puede ser desvinculada de las retribuciones por antigüedad que premian la experiencia laboral. Si tenemos en consideración la nacionalidad de los trabajadores, aquéllos que provienen de países en vías de desarrollo presentan salarios inferiores a la media, que, en el caso de los nativos de África, América del Sur y Centroamérica y los países europeos que no forman parte de la UE15, alcanzan una desventaja del 30\%. Por el contrario, los trabajadores de la UE15 en suelo español superaban este salario medio en un 30\% y los de América del Norte, en un 50\%.

Respecto a las condiciones de entrada en los mercados laborales, hay que señalar que la proporción de mujeres es menor, y ésta se basa principalmente en el sector servicios. De esta manera, la presencia femenina supera escasamente, en 2006, el 40\% (INE, 2007). El protagonismo del sector terciario en los empleos que las mujeres desarrollan o pretenden desarrollar es, de la misma manera, una seña de identidad de los mercados de trabajo en Europa.

En la tabla 2, se puede observar que esta presencia en el mercado de trabajo - ya muy desigual entre hombres y mujeres - se traduce de la misma manera en cuanto al empleo real.

Tabla 2. Estructura del empleo según género y sector de actividad (2006)

\begin{tabular}{lcc}
\hline & Absoluto en miles & Relativo \\
\hline Total & $19.747,7$ & 100 \\
\hline Agrario & 944,3 & 4,78 \\
Industria & $3.292,1$ & 16,67 \\
Construcción & $2.542,9$ & 12,88 \\
Servicios & $12.968,4$ & 65,67 \\
Varones & & \\
\hline Total & $11.742,6$ & 100 \\
\hline Agrario & 681,3 & 5,8 \\
Industria & $2.501,0$ & 21,3 \\
Construcción & $2.408,5$ & 20,51 \\
Servicios & $6.151,7$ & 52,39 \\
Mujeres & & \\
\hline Total & $8.005,1$ & 100 \\
\hline Agrario & 262,9 & 3,28 \\
Industria & 791,1 & 9,88 \\
Construcción & 134,4 & 1,68 \\
Servicios & $6.816,7$ & 85,15 \\
\hline
\end{tabular}

Fuente: datos EPA de 2006 publicados en MTAS (2007) y elaboración propia. 
Las mujeres suponen, escasamente, el $40 \%$ de los trabajadores y, además, el empleo femenino se sitúa de una manera indiscutible en el sector de los servicios, mientras que los hombres están presentes de manera más equilibrada en los demás sectores productivos. Así, las mujeres efectivamente empleadas en 2006 en el sector servicios español suponían más del 85\% del total. En el caso de los hombres, este porcentaje apenas superaba el 50\%, si bien es interesante comentar el gran peso que para los trabajadores masculinos tiene el sector de la construcción. En cuanto al paro, las mujeres vuelven a ser las más damnificadas. En números absolutos, los parados se concentran de manera más evidente en las clases de edad más jóvenes; de todos los parados — hombres y mujeres-, casi un 50\% se encuentra entre los veinte y los treinta y cuatro años; no obstante, hay que tener en cuenta que las diferencias en estas edades entre hombres y mujeres, aunque existentes, no muestran un gran abismo si únicamente tenemos en cuenta la estructura interna del desempleo para cada uno de los grupos. De esta manera, del total de los parados hombres, aquéllos que se encontraban entre los veinte y los treinta y cuatro años representaban un $47,82 \%$, mientras que, en el caso de las mujeres y para este grupo de edad, la cifra que arroja el análisis es de un $47,69 \%{ }^{8}$. Estos datos indican que la juventud es un factor de riesgo para permanecer en situaciones de desempleo, de manera que las consecuencias económicas y sociales que el fenómeno del desempleo provoca se acumulan en las clases de edad más jóvenes.

$\mathrm{Si}$ se tienen en cuenta las tasas de paro (tabla 3) para cada uno de los grupos — hombres y mujeres- y para las diferentes edades, sí hay que dejar claro

Tabla 3. Tasas de paro según sexo y edad (2006)

\begin{tabular}{lccl}
\hline Clases de edad & Total & Hombres & Mujeres \\
De 16 a 19 & $\mathbf{8 , 6}$ & $\mathbf{6 , 4}$ & $\mathbf{1 1 , 6}$ \\
De 20 a 24 & 29,0 & 23,7 & 36,5 \\
De 25 a 29 & 14,8 & 12,3 & 17,8 \\
De 30 a 34 & 10,3 & 8,1 & 12,9 \\
De 35 a 39 & 8,0 & 5,8 & 10,9 \\
De 40 a 44 & 7,2 & 4,9 & 10,2 \\
De 45 a 49 & 6,9 & 4,6 & 9,9 \\
De 50 a 54 & 5,8 & 4,0 & 8,4 \\
De 55 a 59 & 6,1 & 4,0 & 9,5 \\
De 60 a 64 & 6,2 & 5,0 & 8,3 \\
De 65 y más & 4,9 & 4,5 & 5,5 \\
\hline
\end{tabular}

Fuente: datos EPA de 2006 publicados en MTAS (2007) y elaboración propia.

8. Fuente: datos EPA de 2006 publicados en MTAS (2007) y elaboración propia. 
Tabla 4. Temporalidad según sexos (2006)

\begin{tabular}{lcc}
\hline & $\begin{array}{l}\text { Valores absolutos } \\
\text { (en miles) }\end{array}$ & $\begin{array}{l}\text { Valores } \\
\text { relativos }\end{array}$ \\
\hline Total & $\mathbf{1 6 . 2 0 8 , 1}$ & $\mathbf{1 0 0 \%}$ \\
\hline $\begin{array}{l}\text { Contrato indefinido } \\
\text { Contrato de duración determinada }\end{array}$ & $\begin{array}{c}10.691,4 \\
5.516,7\end{array}$ & $35,96 \%$ \\
Varones & & $34,04 \%$ \\
\hline Total & $\mathbf{9 . 3 1 3 , 9}$ & $\mathbf{1 0 0 \%}$ \\
\hline Contrato indefinido & $6.330,1$ & $67,96 \%$ \\
Contrato de duración determinada & $2.983,7$ & $32,04 \%$ \\
Mujeres & & \\
\hline Total & $\mathbf{6 . 8 9 4 , 3}$ & $\mathbf{1 0 0 \%}$ \\
\hline Contrato indefinido & $4.361,3$ & $63,26 \%$ \\
Contrato de duración determinada & $2.533,0$ & $36,74 \%$ \\
\hline
\end{tabular}

Fuente: datos EPA de 2006 publicados en MTAS (2007) y elaboración propia.

que los jóvenes — de ambos sexos — y las mujeres — de cualquier edad — tienen tasas de desempleo superiores a la media, cosa que aumenta la probabilidad del trabajador de formar parte de los llamados ejércitos de reserva o de los segmentos secundarios, inestables y precarios de los mercados laborales. Hay que destacar, como elemento importantísimo de este análisis, que la tasa de paro de las mujeres casi dobla la de los hombres, si tomamos ambos géneros en conjunto.

Respecto al tipo de contrato del que disfrutan los trabajadores, los datos vuelven a mostrar diferencias significativas entre los hombres y las mujeres (tabla 4) en cuanto a la temporalidad.

Y así, en España, cerca de un 68\% de los hombres trabajaban en 2006 con contrato indefinido, frente a un $63,26 \%$ de las mujeres que disfrutaban de estabilidad en el empleo. Además, de entre los más de cinco millones y medio de trabajadores temporales, el 54,08\% estaba constituido por hombres, de manera que las mujeres - que, en general, suponen únicamente 4 de cada 10 empleados- representaban casi el 46\% de los temporales en España en 2006, suponiendo esta realidad un nuevo agravio comparativo. En cuanto a la edad del trabajador (tabla 5), las tasas de temporalidad en los jóvenes superan ampliamente la media y sólo a partir de los treinta años éstas se sitúan en valores de la media e inferiores.

Muestra del grado de problemática que la temporalidad ha alcanzado en España es que, en todo momento, estas tasas son superiores a la media europea. De esta manera, las tasas de temporalidad en España doblan —e incluso mása las europeas. En cualquier caso, parece que las condiciones laborales flexibles afectan de una manera más acusada a las mujeres y a los jóvenes — también 
Tabla 5. Temporalidad según edad del trabajador (2006)

\begin{tabular}{lrrlll}
\hline & $\begin{array}{l}\text { Asalariados, } \\
\text { en miles }\end{array}$ & \multicolumn{1}{c}{ Fijos } & $\begin{array}{l}\text { Porcentaje } \\
\text { del total } \\
\text { de asalariados }\end{array}$ & Temporales & $\begin{array}{l}\text { Porcentaje } \\
\text { del total } \\
\text { de asalariados }\end{array}$ \\
\hline Total & $16.208,1$ & $10.691,4$ & $\mathbf{6 6 , 0}$ & $5.516,7$ & 34,0 \\
\hline De 16 a 19 años & 351,7 & 62,9 & 17,9 & 288,9 & 82,1 \\
De 20 a 24 años & $1.525,6$ & 573,7 & 37,6 & 951,9 & 62,4 \\
De 25 a 29 años & $2.555,6$ & $1.366,5$ & 53,5 & $1.189,2$ & 46,5 \\
De 30 a 39 años & $4.997,3$ & $3.315,6$ & 66,3 & $1.681,7$ & 33,7 \\
De 40 a 49 años & $3.946,7$ & $3.007,4$ & 76,2 & 939,3 & 23,8 \\
De 50 a 59 años & $2.318,6$ & $1.925,2$ & 83,0 & 393,4 & 17,0 \\
De 60 a 69 años & 501,8 & 431,7 & 86,0 & 70,1 & 14,0 \\
De 70 y más años & 10,9 & 8,5 & 78,0 & 2,4 & 21,8 \\
\hline
\end{tabular}

Fuente: datos EPA de 2006 publicados en MTAS (2007) y elaboración propia.

a los inmigrantes, para quienes esta tasa alcanzaba un 63\% en 2003 (Izquierdo, 2005) — que a los hombres y adultos. Teniendo también en cuenta que las tasas de paro son superiores para las mujeres y los jóvenes, se puede empezar a efectuar un primer esbozo de los segmentos precarios.

Respecto a las jornadas parciales (tabla 6), la realidad muestra que son ocupadas por mujeres en casi un $80 \%$ de los casos. Pero es cuando pasamos a los motivos que llevan al trabajador a desarrollar una actividad a tiempo parcial cuando se puede observar el enorme peso que todavía tienen los factores relativos al tradicional papel de la mujer como miembro de la familia encargado de las tareas reproductivas, a la hora de impedir o dificultar el pleno acceso femenino al mundo del trabajo.

De esta manera, las principales motivaciones del hombre para trabajar a tiempo parcial son que o bien no encontró otro tipo de empleo - el 32\% de los trabajadores-, o bien necesitaba la jornada parcial para compatibilizarla con actividades de formación - más de un $24 \%$ de los ocupados a tiempo parcial- . En el caso de las mujeres, este interés por la formación afecta sólo a un $9 \%$ de las trabajadoras, y así, para la mujer, los principales motivos son que no encontró otro tipo de empleo — con un valor del 32,3\% - y que debían compatibilizarlo con obligaciones de tipo familiar, razón ésta que esgrimían el $30,7 \%$ de las trabajadoras con contrato a tiempo parcial frente a un reducido $3,5 \%$ en el caso de los hombres. La precariedad en este tipo de contratos debemos asociarla a dos grandes razones: la primera, la no-voluntariedad en el caso de dos tercios de las mujeres, que se ven obligadas a trabajar de esta manera aunque preferirían disponer de una jornada completa; la segunda, que, al trabajar menos horas, perciben salarios menores y, por lo tanto, se pone en cuestión la independencia económica de quien realiza un empleo bajo este tipo de relación contractual. 
Tabla 6. Jornada parcial según género y motivos

\begin{tabular}{|c|c|c|c|c|c|c|}
\hline & \multicolumn{2}{|l|}{ Total } & \multicolumn{2}{|c|}{ Hombres } & \multicolumn{2}{|l|}{ Mujeres } \\
\hline & \multicolumn{2}{|c|}{$\overline{\text { Valores absolutos }}$} & \multicolumn{2}{|c|}{$\overline{\text { Valores absolutos }}$} & \multicolumn{2}{|c|}{ Valores absolutos } \\
\hline & en miles & $\%$ & en mile & $\%$ & en miles & $\%$ \\
\hline Total & $2.361,3$ & 100,0 & 507,8 & 100,0 & $1.853,5$ & 100,0 \\
\hline $\begin{array}{l}\text { No encontró } \\
\text { a tiempo completo }\end{array}$ & 759,8 & 32,2 & 161,8 & 31,9 & 598,1 & 32,3 \\
\hline $\begin{array}{l}\text { No quiso } \\
\text { a tiempo completo }\end{array}$ & 260,6 & 11,0 & 46,8 & 9,2 & 213,8 & 11,5 \\
\hline Asistencia a cursos & 290,8 & 12,3 & 125,7 & 24,7 & 165,1 & 8,9 \\
\hline $\begin{array}{l}\text { Enfermedad } \\
\text { o incapacidad }\end{array}$ & 44,6 & 1,9 & 19,6 & 3,9 & 25,0 & 1,3 \\
\hline Obligaciones familiares & 586,5 & 24,8 & 17,8 & 3,5 & 568,7 & 30,7 \\
\hline Otros motivos & 404,4 & 17,1 & 132,3 & 26,1 & 272,1 & 14,7 \\
\hline No sabe el motivo & 14,6 & 0,6 & 3,8 & 0,8 & 10,7 & 0,6 \\
\hline
\end{tabular}

Fuente: datos EPA de 2006 publicados en MTAS (2007) y elaboración propia.

Siguiendo este argumento, los hombres y las mujeres no acceden a los mismos tipos de empleos (tabla 7), y se puede observar un mayor número de mujeres en empleos que, en principio, van a estar peor retribuidos (tabla 8). Efectivamente, las mujeres estarán especialmente subrepresentadas en la mayor parte de las actividades industriales y en algunos servicios auxiliares a estas industrias. Por el contrario, se encontrarán sobrerrepresentadas principalmente en la hostelería, en el comercio al por menor, en actividades sanitarias y de servicios sociales, en educación, en servicios personales y en servicios domésticos y actividades asociativas, recreativas y culturales, todas ellas actividades terciarias. Respecto al sector industrial, sólo en la rama de industrias textiles y de la confección las mujeres tienen una presencia mayoritaria. La gran mayoría de estas actividades — con la excepción de, por ejemplo, las profesiones educativas- tienen asociados sueldos menores — gasto salarial por hora trabajada - al 90\% de la media, que se situaba en 2006 en 10,30 euros la hora.

Muchas de ellas tienen desventajas de alrededor de un $20 \%$ o más con esta media, como, por ejemplo, la industria textil, que no alcanza el $85 \%$ del sueldo medio; la hostelería, que se queda en un $70 \%$, y el comercio al por menor, que no supera el $73 \%$. Es necesario destacar dos actividades: por un lado, la construcción - que aunque ocupa mayoritariamente a hombres, tiene valores de, únicamente, un $88 \%$ del sueldo mediograndes motores de empleo en la España actual y es, además, un sector en el que trabajan muchos de los recién llegados a nuestro país, como veremos más adelante. Por otro lado, los servicios personales, otro sector en el que se están empleando masivamente trabajadores extranjeros — trabajadoras, más bien- 
Tabla 7. Sectores y ramas de actividad (selección) según géneros (2006)

\begin{tabular}{|c|c|c|c|c|c|}
\hline & Total & $\begin{array}{l}\text { Hombres } \\
\text { En miles }\end{array}$ & $\%$ & $\begin{array}{l}\text { Mujeres } \\
\text { En miles }\end{array}$ & $\%$ \\
\hline Total & $\overline{12.990,5}$ & $7.720,4$ & 59,4 & $5.270,2$ & 40,6 \\
\hline \multicolumn{6}{|l|}{ Sectores } \\
\hline Industria & $2.380,8$ & $1.790,1$ & 75,2 & 590,6 & 24,8 \\
\hline Construcción & $1.883,6$ & $1.714,5$ & 91,0 & 169,0 & 9,0 \\
\hline Servicios & $8.726,2$ & $4.215,7$ & 48,3 & $4.510,5$ & 51,7 \\
\hline \multicolumn{6}{|l|}{ Ramas } \\
\hline Industria de alimentos, bebidas y tabaco & 322,9 & 198,7 & 61,5 & 124,2 & 38,5 \\
\hline Industria textil y de la confección & 158,0 & 60,3 & 38,1 & 97,7 & 61,9 \\
\hline Industria del cuero y del calzado & 37,7 & 22,9 & 60,8 & 14,8 & 39,2 \\
\hline Industria química & 140,9 & 96,3 & 68,3 & 44,6 & 31,7 \\
\hline Metalurgia & 60,6 & 54,6 & 90,2 & 6,0 & 9,8 \\
\hline Construcción, maquinaria y equipo mecánico & 159,7 & 138,5 & 86,7 & 21,2 & 13,3 \\
\hline Fabricación de automóviles y remolques & 167,4 & 140,6 & 84,0 & 26,8 & 16,0 \\
\hline Construcción & $1.883,5$ & $1.714,5$ & 91,0 & 169,0 & 9,0 \\
\hline Comercio al por mayor. Interm. del comercio & 875,7 & 574,4 & 65,6 & 301,4 & 34,4 \\
\hline Comercio al por menor. Reparac. domésticas & $1.406,8$ & 537,9 & 38,2 & 868,9 & 61,8 \\
\hline Hostelería & 839,8 & 375,3 & 44,7 & 464,5 & 55,3 \\
\hline Instituciones financieras y seguros & 405,0 & 235,1 & 58,1 & 169,9 & 41,9 \\
\hline Inmobiliarias. Alquiler de bienes muebles & 262,4 & 134,5 & 51,2 & 127,9 & 48,8 \\
\hline Educación & 516,6 & 187,9 & 36,4 & 328,8 & 63,6 \\
\hline Activ. sanitarias, veterinar. y Serv. sociales & 959,0 & 250,2 & 26,1 & 708,9 & 73,9 \\
\hline Activ. div. serv. pers. y pers. Doméstico & 220,8 & 67,8 & 30,7 & 153,0 & 69,3 \\
\hline
\end{tabular}

Fuente: datos de la Encuesta de Coyuntura Laboral, consultados en MTAS (2006) y elaboración propia.

y que tiene el coste salarial más bajo (6,32 euros por hora), que supone únicamente un $61 \%$ del coste salarial medio en 2006.

Pero estos sectores con peores salarios no están únicamente ocupados por mujeres. Los inmigrantes, entendidos como otro de los colectivos débiles en los mercados de trabajo, también logran su acceso al empleo en sectores o ramas de actividad que a duras penas ofrecen buenas retribuciones. De esta manera, los inmigrantes en $2006^{9}$ han conseguido permisos de trabajo principalmente para las siguientes actividades: hogares que emplean personal doméstico, hostelería, construcción y agricultura, destacando la construcción como

9. Fuente: datos del Ministerio de Administraciones Públicas, elaborados por la Subdirección General de Estadísticas Sociales y Laborales, consultado en MTAS (2007). 
Tabla 8. Coste salarial para algunas ramas seleccionadas de actividad (2006)

\begin{tabular}{lcc}
\hline & $\begin{array}{c}\text { Coste salarial } \\
\text { en euros }\end{array}$ & $\begin{array}{c}\text { Puntuación relativa } \\
\text { si la media és índice 100 }\end{array}$ \\
\hline Total & 10,30 & 100 \\
\hline Sectores & & \\
Industria & 11,20 & 108,74 \\
Construcción & 9,11 & 88,45 \\
Servicios & 10,32 & 100 \\
Ramas seleccionadas & & \\
\hline Industria de alimentos y bebidas & 9,72 & 94,37 \\
Industria textil & 8,72 & 84,66 \\
Industria de la confección & 7,07 & 68,64 \\
Industria del cuero y del calzado & 7,49 & 72,72 \\
Industria química & 14,55 & 141,26 \\
Metalurgia & 14,33 & 139,13 \\
Construcción maquinaria y equipo mecánico & 11,61 & 112,72 \\
Fabricación de automóviles y remolques & 13,71 & 133,11 \\
Reciclaje & 9,08 & 88,16 \\
Construcción & 9,11 & 88,45 \\
Comercio al por mayor. Interm. del comercio & 9,94 & 96,5 \\
Comercio al por menor. Reparac. domésticas & 7,50 & 72,82 \\
Hostelería & 7,31 & 70,97 \\
Instituciones financieras excepto seguros & 19,77 & 191,94 \\
Actividades inmobiliarias & 9,50 & 92,23 \\
Educación & 14,20 & 137,86 \\
Activ. sanitarias y veterinarias, serv. sociales & 13,59 & 131,94 \\
Actividades diversas de servicios personales & 6,32 & 61,36 \\
\hline
\end{tabular}

Fuente: datos del INE relativos al Índice de Costes Laborales, consultados en MTAS (2007) y elaboración propia.

gran empleadora de hombres, y el servicio doméstico, de mujeres - casi la mitad de los permisos de trabajo que se expiden para mujeres en 2006 es para desarrollar un empleo en este ámbito-, si bien hay también un gran porcentaje de hombres que encuentra empleo en este sector.

En cuanto a los inmigrantes que ya trabajan en España ${ }^{10}$, cabe decir que, en 2006, éstos suponían un 9,7\% del total de los trabajadores. Pero para determinadas actividades, como la construcción o la agricultura, este porcentaje alcanza el $15 \%$ del total de los trabajadores, el $20 \%$ en la hostelería y cerca del $60 \%$ del total de los trabajadores en los hogares que emplean personal doméstico. De nuevo se puede observar una sobrerrepresentación de colectivos débiles de trabajadores en empleos con menores retribuciones.

10. Fuente: datos de la Dirección General de la Policía del Ministerio de Interior, tratados por el Observatorio Permanente de la Inmigración, del Ministerio de Trabajo y Asuntos Sociales, consultados en MTAS (2007). 
Tabla 9. Accidentes de trabajo según gravedad y tipo de contrato (2006)

\begin{tabular}{lcccccccc}
\hline & Totales & $\%$ & \multicolumn{1}{c}{ Leves } & $\%$ & Graves & $\%$ & \multicolumn{2}{c}{ Mortales $\%$} \\
\hline Total & 911.561 & 100 & 902.062 & 100 & 8.552 & 100 & 947 & 100 \\
\hline Contratos indefinidos & 422.101 & 46,31 & 418.086 & 46,34 & 3.528 & 41,25 & 487 & 51,42 \\
Contratos temporales & 479.206 & 52,57 & 474.144 & 52,56 & 4.622 & 54,05 & 440 & 46,46 \\
No clasificables & 10.254 & & 9.832 & & 402 & & 20 & \\
\hline
\end{tabular}

Fuente: datos elaborados por la Subdirección General de Estadísticas Sociales y Laborales, consultados en MTAS (2006) y elaboración propia.

Por último, es necesario considerar los accidentes de trabajo y las enfermedades profesionales como el elemento extremo de la precariedad laboral. Cuando se produce un accidente laboral o cuando un trabajador enferma debido a las condiciones en las que realiza su tarea, y más si este accidente o esta enfermedad tienen consecuencias graves para su salud, la peligrosidad o la dureza de determinados empleos se hacen visibles de manera trágica. Y se hacen visibles no sólo las características intrínsecas de algunos empleos — que pueden suponer situaciones de riesgo más o menos calculado para la integridad física o psíquica del trabajador-, sino también los límites que la desregulación y la flexibilidad imponen a algunos empleos en forma de ritmos de trabajo más elevados, reducción hasta la anécdota de los períodos de formación, ahorro en dispositivos de seguridad y/o protección, etc. En la tabla 9, se consideran los accidentes de trabajo según su gravedad y según el tipo de contrato del accidentado. Se observa una relativa igualdad - los porcentajes son sólo ligeramente superiores al 50\% en los trabajadores con contrato inestable - en los porcentajes de los accidentados, tanto si el accidente es leve, grave o mortal - en este caso, los trabajadores temporales están ligeramente por debajo del $50 \%$ - ; pero hay que tener en cuenta que estos trabajadores sólo suponen el $35 \%$ del total de los asalariados, de forma que la siniestralidad afecta de manera más evidente al colectivo de temporales, donde las mujeres, los jóvenes y los inmigrantes se encuentran presentes de manera rotunda.

Es muy interesante, en este sentido, valorar de nuevo el sector de la construcción, donde hay un mayor número de inmigrantes y donde se concentran algunas de las actividades con mayor riesgo para la salud. Efectivamente, en este sector, el porcentaje de accidentes leves, graves y, sobretodo, mortales es bastante importante respecto al peso real del sector en la estructura productiva. De hecho, el 33\% de los accidentes mortales en 2006 han sucedido en el sector de la construcción (MTAS, 2007). Respecto a las enfermedades profesionales ${ }^{11}$, el sector productivo que se encuentra sobrerrepresentado es, en general, el de la industria, de manera que una de cada dos enfermedades deri-

11. Datos elaborados por la Subdirección General de Estadísticas Sociales y Laborales, consultados en MTAS (2006). 
vadas de la actividad laboral se producen en actividades industriales, hecho que debe estar relacionado con el manejo de materiales tóxicos, el disponer de un ambiente de trabajo poco saludable o cualquier otro factor que pueda desencadenar una dolencia relacionada con el trabajo.

Tanto en cuanto a los accidentes de trabajo como respecto a las enfermedades profesionales - y a expensas de una valoración más exhaustiva según las diferentes ramas de actividad-, hay que destacar que los sectores de servicios se encuentran con porcentajes de siniestralidad relativamente menores que en otros sectores productivos y, en general, por debajo de lo que correspondería de acuerdo con el peso que alcanza en la estructura productiva del sistema económico español.

\section{Conclusiones. Nuevas clases trabajadoras, nueva estructura social}

Durante el texto, se ha hecho mención del importante crecimiento del sector servicios en los países desarrollados y de las oportunidades de empleo que éste ofrece. En este sentido, cabe también mencionar (Anxo y Storrie, 2001) que estos sectores económicos seguirán siendo en el futuro los mayores generadores de empleo. Pero esta terciarización ha venido acompañada de un cambio en el modelo de empleo que produce, de acuerdo con los datos que se han expuesto, una cierta precarización del empleo, tanto a nivel europeo como a nivel español, y que centra sus efectos, de manera especial, en los colectivos de mujeres e inmigrantes. La emergencia de nuevos empleos o el crecimiento de éstos ha generado también la emergencia de nuevos trabajadores que buscan consolidarse en los mercados de trabajo, a pesar de que las oportunidades laborales no ofrecen estabilidad laboral ni condiciones económicas ventajosas.

Así, en este nuevo modelo de empleo en servicios, conviven oportunidades laborales en las que los trabajadores disponen de condiciones objetivas de empleo beneficiosas, por ejemplo, en cuanto a salarios o en cuanto a flexibilidad, que permiten la conciliación de la vida familiar y profesional, con otras oportunidades que se caracterizan por condiciones de trabajo más complicadas. Los colectivos de trabajadores que están afectados en mayor medida por esta precariedad, los segmentos secundarios de mercado de trabajo, son las nuevas clases trabajadoras del sistema productivo y del modelo de acumulación que emerge a partir de las crisis de los años setenta y del final del modelo fordista y de la norma social del empleo estable. En este sentido, las transformaciones que han sido efectivas sobre la fuerza de trabajo hacen que ésta tome formas diferentes a la tradicional clase obrera, de manera que podríamos considerar que existe una nueva clase obrera que convive junto a la clase obrera industrial o que, en una interpretación más conservadora, la clase obrera de hoy en día es mucho más heterogénea y, aunque sigue incluyendo elementos que desarrollan su actividad laboral en los sectores industriales, ahora el contingente mayor se encuentra trabajando en los servicios. En este sentido, las actividades terciarias toman el protagonismo en cuanto al número de asalariados en general, y esto puede representar que se consolide la mutación de la clase obrera tradicional, reali- 
dad ésta que debe afectar al conjunto de la estructura social de los países desarrollados. En resumen, la situación actual es de una profunda aceleración en los procesos de cambio, tanto en cuanto a las estructuras productivas y a los modelos de acumulación, como en cuanto al mercado de trabajo, que debe adaptarse a nuevos ritmos productivos y a nuevas fórmulas o formas de producir. De esta manera, las nuevas clases trabajadoras deben seguir los ritmos que se marcan desde las necesidades productivas de bienes y servicios, y esto puede tener consecuencias directas en la estructura social de las sociedades salariales de servicios.

El trabajo, en definitiva, como fuente principal de rentas en las sociedades desarrolladas, tiene una traducción inmediata en términos económicos y de capacidad de consumo. Pero no es sólo el salario lo que determina la clase social, ya que el tipo de trabajo o el sector en el que se desarrolla éste es un elemento también importante para considerar esta realidad. De esta manera, puede concluirse que determinadas ocupaciones de servicios, incluso en subsectores prestigiados, pero especialmente en aquellas actividades donde es necesario un cierto grado de intensidad en el factor trabajo - hostelería, comercio, servicios personales-, no se adecuan a lo establecido para las clases medias ni para la tradicional clase obrera industrial.

Sabemos, así, que el crecimiento de todos estos sectores genera una importante masa de trabajadores que no están considerados dentro de la clase obrera tradicional, pero también que los orígenes de estos trabajadores, los colectivos de los cuales proceden, sus expectativas y sus necesidades son diferentes, y todo esto podría ser fruto de los procesos de fragmentación social y de segmentación de los mercados de trabajo, en este sentido:

Lo relevante será, cada vez en mayor grado, el tipo concreto de trabajo o actividad que cada cual realice y las condiciones en las que lo haga, con mayor o menor seguridad y formalización, con mejor o peor remuneración, con mayor o menor comodidad, etc. Y, sobre todo, lo más decisivo serán las oportunidades de desarrollar una trayectoria laboral integrada [...]. Por ello, los grandes alineamientos sociales y las grandes fracturas desigualitarias y de postergación social en las nuevas sociedades tecnológicas presentarán varias líneas cruzadas, que darán lugar a que finalmente se sitúen a un mismo lado de las fronteras sociales personas de distinta trayectoria y procedencia (Tezanos, 2001: 286).

Así, los nuevos puestos de trabajo podrían no necesitar de la clase obrera tradicional para reclutar a los nuevos empleados, de manera que concluir que éstos son clase obrera puede presentar dificultades, pero defiendo que las nuevas clases trabajadoras, que incluyen a muchos de los trabajadores terciarios y en las que hay una gran presencia de mujeres e inmigrantes, tienen también una base objetiva y real en cuanto a condiciones laborales y de vida que, fuera de las identificaciones entre los empleados que pueden tener lugar, supone una existencia real como colectividad, no sólo en el mundo del trabajo, sino también a nivel social. Lo que sucede es que las características de precariedad de este nuevo empleo dificultan la participación social de los individuos que lo 
sufren y transforman la inseguridad laboral de estos trabajadores en inseguridad social (Alonso, 2007) a través de la vida familiar y personal de los individuos. Los ámbitos del trabajo y de la ciudadanía, a pesar de una aparente separación que tiene su razón de ser, precisamente, en el menor valor de la ocupación para muchos individuos y grupos sociales, están unidos en el momento en el que, para desarrollarse en uno, se necesitan los ingresos que se consiguen en el otro.

$\mathrm{Y}$, frente a esta realidad, las nuevas clases trabajadoras, que se enfrentan a condiciones de trabajo duras y a escasas o insuficientes retribuciones, como hemos visto al considerar el caso de los working poor, no disponen todavía de la capacidad de resistencia y organización de las clases obreras tradicionales, debido, en parte, a la heterogeneidad social existente en los segmentos secundarios del mercado de trabajo, a la incapacidad o imposibilidad de los sindicatos y organizaciones obreras de consolidarse entre estos trabajadores y a la propia dinámica de las relaciones laborales modernas en las que se tiende más hacia el establecimiento de relaciones individuales entre el trabajador y la dirección o gestión empresarial que hacia la negociación colectiva, de manera que la identificación entre trabajadores que desarrollan las mismas actividades se complica. En este sentido, es interesante considerar la doble vertiente (Miguélez, 2004) de las nuevas estrategias de flexibilización empresariales que, por una parte, se dirigen al ahorro en costes salariales y, por tanto, hacia una mayor rentabilidad económica y, por la otra, hacia el disciplinamiento de los mercados laborales a través del control de los propios trabajadores y de las organizaciones sindicales, que ven moderadas o dificultadas sus reivindicaciones.

\section{Bibliografía}

Alonso, L. E. (2007). La crisis de la ciudadanía laboral. Barcelona: Anthropos.

ANXO, D. y STORRIE, D. (eds.) (2001). The job creation potential of the service sector in Europe. Luxemburgo: European Commission. Office for Official Publications of the European Communities.

BAUMAN, Z. (2001). Globalització: Les conseqüències humanes. Barcelona: Universitat Oberta de Catalunya.

BiLBAO, A. (1999). El empleo precario: Seguridad de la economía e inseguridad del trabajo. Madrid: Los Libros de la Catarata.

BraVERMAN, H. (1974). Labor and monopoly capital. The degradation of work in the twentieth century. Nueva York: Monthly Review Press.

Bueno Campos, E. (2002). «Globalización, sociedad red y competencia. Hacia un nuevo modelo de empresa». Revista de Economía Mundial, 7, 23-37.

CASTEL, R. (1997). La metamorfosis de la cuestión social. Buenos Aires: Paidós.

CAstells, M. (1997). La era de la información. Economía, sociedad, cultura, vol. 1. Madrid: Alianza.

EUROPEAN FOUNDATION FOR THE IMPROVEMENT OF LIVING AND WORKING CONDITIONS (EUROFUND) (2004). Working poor in the European Union. Luxemburgo: Office for Official Publications of the European Communities. 
- (2005). Fifteen years of working conditions in the EU: Charting the trends. Dublín: EUROFUND.

- (2007). Annual Review of Working Conditions in the EU 2006-2007. Luxemburgo: Office for Official Publications of the European Communities.

García Nogueroles, J. M. (2009). «Segmentación, precariedad y nueva ciudadanía: Consecuencias de los cambios en el modelo de empleo". Aposta: Revista de Ciencias Sociales, 41, abril, mayo, junio [en línea]. <http://www.apostadigital.com/ revistav3/hemeroteca/jmnoguer2.pdf>.

GORDON, D.; EDWARDS, R.; REICH, M. (1986). Trabajo segmentado, trabajadores divididos: La transformación histórica del trabajo en los Estados Unidos. Madrid: Ministerio de Trabajo y Seguridad Social.

INE (2004). Encuesta de estructura salarial 2002. Principales resultados. Madrid: INE [en línea]. <http://www.ine.es/daco/daco42/salarial/prinre02.pdf>.

- $(2007,2009)$. Instituto Nacional de Estadistica [en línea]. <http://www.ine.es>.

IZQUIERDO, A. (2005). "Claroscuros de la inmigración en España». Inmigración, enero, cuarta etapa.

Kern, H. y SCHUMANn, M. (1988). El fin de la división del trabajo: Racionalización en la producción industrial. Madrid: Ministerio de Trabajo y Seguridad Social.

LÓPEZ ROLDÁN, P. (1996). "La construcción de una tipología de segmentación del mercado de trabajo». Papers: Revista de Sociologia, 48, 41-58.

López Roldán, P.; Miguélez, F.; Lope, A. y Coller, X. (1998). «La segmentación laboral: hacia una tipología del ámbito productivo». Papers: Revista de Sociología, 55, 45-77.

MARSHALl, A. (1992). «Secuelas del paro: el nuevo papel del trabajo temporal y del trabajo a tiempo parcial en Europa Occidental». En: El trabajo precario en la regulación del mercado laboral: Crecimiento del empleo atipico en Europa Occidental. Madrid: Ministerio de Trabajo y Seguridad Social.

MedialdeA, B. y Álvarez, N. (2005). «Ajuste neoliberal y pobreza salarial: Los working poor en la Unión Europea». Viento Sur, 82, septiembre, 56-64.

MiguÉLEZ, F. (2002). «¿Por qué empeora el empleo?». Sistema, 168-169, 37-52.

- (2004). «La flexibilidad laboral». Trabajo: Revista Andaluza de Relaciones Laborales, $13,17-36$.

- (2006). «Economía social y empleo: El caso de España». Papers: Revista de Sociologia, 81, 11-36.

- (2007). «Flexiseguridad, bienestar y cohesión social». Revista del Ministerio de Trabajo y Asuntos Sociales, núm. extra 1, 145-159.

MTAS (2007). Anuario de Estadísticas Laborales y de Asuntos Sociales 2006. CD-ROM. Madrid: Ministerio de Trabajo y Asuntos Sociales [en línea]. <http://www.mtas.es/estadisticas/anuario2006/welcome.htm>.

PRIETO, C. (2007). «Del estudio del empleo como norma social al de la sociedad como orden social». Papeles del CEIC, vol. 2007/1, 28. Universidad del País Vasco. CEIC (Centro de Estudios sobre la Identidad Colectiva) [en línea]. <http://www.ehu.es/CEIC/pdf/28.pdf>.

ReCiO, A. (2001). «Una nota sobre bajos salarios en España». Cuadernos de Relaciones Laborales, 18, 15-45.

REGNARD, P. (2007). «Minimum wages 2007. Variations from 92 to 1570 euro gross per month». EUROSTAT. Statistics in Focus, 71/2007 [en línea]. <http://epp.eurostat. ec.europa.eu/cache/ITY_OFFPUB/KS-SF-07-071/EN/KS-SF-07-071-EN.PDF>.

TEZANOS, J. F. (2001). La sociedad dividida: Estructuras de clases y desigualdades en las 
sociedades tecnológicas. Madrid: Biblioteca Nueva.

US DEPARTMENT OF LABOR (2006). A profile of the working poor, 2004 Report 994. US Department of Labor. US Bureau of Labor Statistics, may [en línea]. $<$ http://www.bls.gov/cps/cpswp2004.pdf>.

VILLA, P. (1990). La estructuración de los mercados de trabajo: La siderurgia y la construcción en Italia. Madrid: Ministerio de Trabajo y Seguridad Social. 